\title{
SKIN MANIFESTATIONS OF THE RETICULOSES
}

\author{
By F. Ray Bettley, F.R.C.P. \\ Physician for Diseases of the Skin, The Middlesex Hospital, London
}

The conception of the reticulo-endothelial system belongs to the realm of experimental pathology. The reticuloses, a group of disorders primarily affecting that system are, therefore, a concept of pathology and their clinical manifestations must be diverse. Indeed, even among pathologists, opinions are divided as to the criteria which govern admission to the group. It would be widely conceded that reticuloses are liable to involve cells of the reticulo-endothelial system in whatever part of the body they occur, and since the skin is a very readily observed organ in which reticulo-endothelial elements are numerous, it is not surprising that most reticuloses have dermatological manifestations-though some much more frequently than others.

At the present time the reticulosis group is made up of a number of syndromes which in their dermatological manifestations are characterized by a massive infiltration of the dermis with cells of proved or presumed origin from the reticuloendothelial system. As Goldsmith (1944) pointed out, it would be absurd to make the condition that the cause must be unknown, i.e. that no infective or similar agent has been identified. At the same time, it is impossible entirely to escape the notion that the chronic granulomatous process of Hodgkin's disease is acceptable as a reticulosis, whereas to include the widespread chronic granulomatoses of the well-known infections would be to broaden the concept of reticulosis until it loses its meaning. With very few exceptions then, the causes of the reticuloses are unknown. To the clinician a purely pathological classification is not entirely satisfactory; a purely clinical classification is still more unsatisfactory since a single entity such as mycosis fungoides may produce clinical manifestations of great diversity. These manifestations may be grouped into three classes and it is from this purely clinical aspect that the reticuloses are now considered; such a clinical classification cuts across the pathological classification and is in no way a substitute for it. The three types of manifestation referred to are: (I) Generalized pruritus; (2) superficial eruptions; and (3) infiltrated or tumour-like lesions.

\section{Generalized Pruritus}

The generalized pruritus which occurs in the reticuloses is most often seen in Hodgkin's disease and mycosis fungoides, and less often in lymphatic $\frac{\overline{\bar{p}}}{\frac{\bar{c}}{2}}$ leukaemia. The irritation usually affects equally $\underset{\mathbb{}}{\mathscr{Q}}$ the entire skin surface and is at first unaccompanied by any objective change in the skin. It is a severe $\overrightarrow{0}$ itching, sometimes paroxysmal, but seldom re- $?$ mitting completely. This severe irritation usually $\vec{\omega}$ leads to violent scratching, and the secondary effects of excoriation and lichenification of the skin 8 may develop after a few weeks. The riails are often 3 . polished by their friction against the skin in the act of scratching.

During the phase of general pruritus other 8 manifestations of the reticulosis may not be identifiable and even biopsy of the skin may show ${ }_{0}^{N}$ no characteristic changes and give no help in $\frac{}{r}$ diagnosis. Indeed, the phase of general pruritus $\overrightarrow{ }$ often ceases as the reticulosis evolves, so that by $\subsetneq$ the time diagnosis is possible, the irritation has already disappeared. The differential diagnosis of $\vec{c}$ generalized pruritus may, therefore, be extreme difficult or impossible. Conditions such jaundice, senile pruritus, metabolic upsets such those associated with uraemia and, of course, parasitic skin diseases must all be excluded. General pruritus of psychiatric origin may be difficult to distinguish and often, when clinical examination reveals no cause and pathological tests give no help, the nature of this symptom can only be recognized after a period of observation during which some other manifestation of the reticulosis develops.

\section{Superficial Eruptions}

In this category are included a wide diversity of $\delta$ eruptions in which the dermis, and often the epidermis, are involved without any notable degree 은 of infiltration in the lesions. Sometimes an $D$ eruption of this kind follows a period of general pruritus; in other cases a superficial eruption gradually becomes more infiltrated until it passes into the third category of tumour-like lesions.

It is in mycosis fungoides that this class of eruption is most commonly and most typically seen. The disease often starts with a widespread eruption 6 affecting principally the trunk and proximal parts of the limbs. The rash is usually made up of large erythematous sheets, profusely scaly, itchy and sometimes weeping. In this way eczema and psoriasis may be simulated for a time. Characteristic of these eruptions, however, is a blotchy 
brick-red or dusky colour which often gives a clue to diagnosis. Biopsy of the skin may show pathognomonic changes but in other cases gives no help. This stage of erythroderma may be of short or of comparatively long duration, occasionally lasting for many years before the tumour stage of the disease is reached.

In other cases a widespread or, more often, generalized eczematous eruption, the appearances of which seem to be typical of eczema or seborrhoeic dermatitis, is eventually followed by the development of a reticulosis (Fig. I). In these cases, early diagnosis may be particularly difficult, since the eruption is identical in appearance with other cases of eczema or seborrhoeic dermatitis which eventually clear up without any sign of reticulosis.

Exfoliative dermatitis, a result of widespread eczema, seborrhoeic dermatitis or psoriasis, usually loses the characteristics of the original lesion, and whatever their origin, such cases occasionally pass into reticulosis. This is often taken to indicate that the skin eruption was really from the outset a manifestation of the reticulosis; it is pertinent, however, to ask whether reticulosis may not arise as a consequence of long-standing erythroderma. Nearly always, in such cases, the reticulosis appears only after the skin changes have continued for some years, or even decades.

These cases should be distinguished from the so-called lipo-melanic reticulosis (Pautrier and Woringer, 1932), in which a special type of lymphadenopathy occurs. This lymphadenopathy, characterized by considerable accumulation of monocytes and the presence of melanin and fat in the lymph nodes, may complicate a variety of widespread dermatoses. There can be little doubt that it is really a rather special type of non-specific lymph gland response which disappears once the skin lesions heal. This type of lymphadenopathy should, therefore, not be classed with the true reticuloses and it is misleading to include it in this group.

In recent years it has been observed that several uncommon chronic dermatoses occasionally pass on to frank and apparently typical mycosis fungoides. Parapsoriasis en plaques is a symptomless extremely chronic eruption made up of welldefined pink macules covered with fine scaling and with only the slightest degree of cellular infiltration in the dermis either clinically or histologically. Both this condition and poikoloderma are sometimes seen to proceed to mycosis fungoides, though in most cases such an evolution does not occur.

Urticarial eruptions are occasionally seen in other reticuloses; the vesicular leucaemide, consisting of a widespread eruption of small isolated vesicles on a slightly infiltrated base, is a rare curiosity.

Acquired ichthyosis, or xeroderma, is occasionally seen; the whole skin surface becomes dry and is covered with fine adherent scales. Itching is slight or absent. This change in the skin is usually a late manifestation and is often associated with cachexia (Fig. 2). It is presumably metabolic in origin, since the histology of the skin shows no abnormal cells.

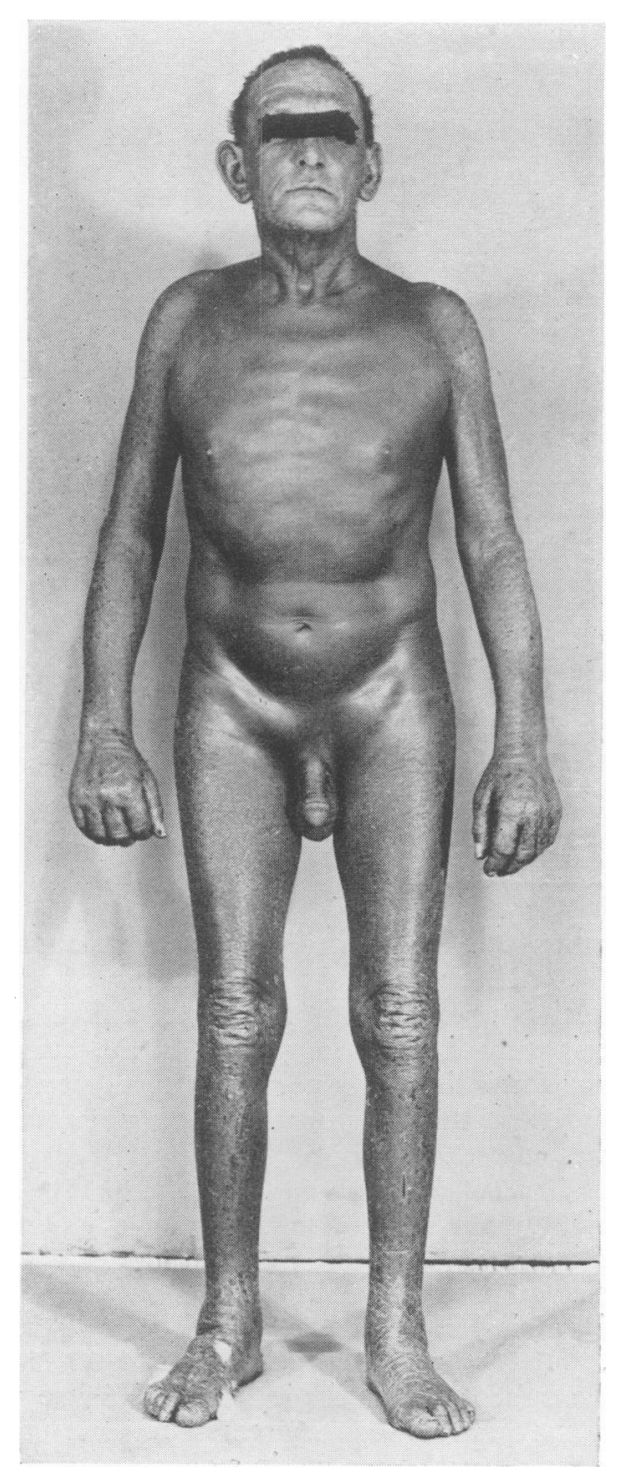

FIG. I. - Longstanding generalized erythroderma ('Homme Rouge'). The entire surface is scaly and red. Note the enlargement of superficial lymph glands. (Dr, H. T. H, Wilson's case.) 


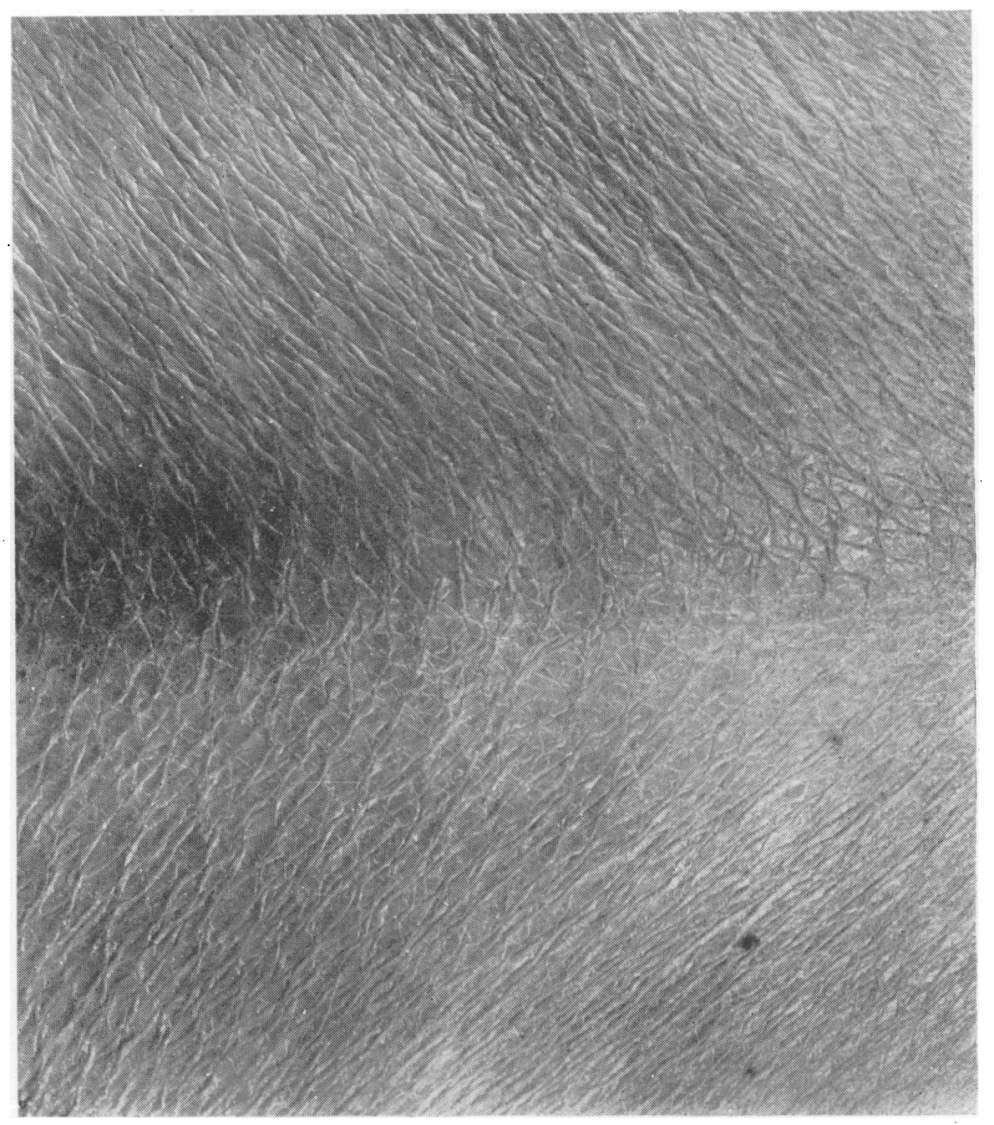

Fig. 2.-Acquired xeroderma. Section of the midline of the back in a patient with advanced Hodgkin's disease.

\section{Tumours}

Tumours and infiltrated lesions are encountered in all gradations, from a slightly infiltrated plaque to massive tumours up to the size of a melon. In mycosis fungoides it is usual for the stage of tumour formation to supervene after a period of a few years, during which superficial eruptions appear. Less commonly, however, is seen the type à tumeurs d'emblée, in which the formation of dermic tumours is the first sign of the disease. In either type the tumours are situated in the dermis, starting as deep red or purplish, firm hemispherical swellings about half-an-inch across (Fig. 3). The lesions are usually freely mobile on the subcutaneous tissue and remain so even when they have much increased in size. They grow rapidly and often attain a diameter of two or three inches in the course of a month. By the time they are this size, the mass often shows some lobulation and resembles a half tomato stuck on the surface of the skin. The epidermis is at first stretched tightly over the tumour, but eventually ulcerates. A good deal of necrosis often results, leaving a deep punched-out ulcer surrounded by a thick rim of tumour tissue. It is a remarkable characteristic of these tumours that they may involute spontaneously at any time and heal as rapidly as they appear, with little or no scarring. As the disease progresses, however, more and more tumours develop and the tendency to spontaneous involution is reduced until the patient may be covered with as many as 50 ulcerated tumours varying up to several inches across (Fig. 4).

The development of cutaneous tumours in Hodgkin's disease is decidedly less common. When they do appear, they are often the first sign 0 of the disease, and a meticulous search may reveal no other manifestations for a time. In due course, however, the more common manifestations develop and the disease runs an otherwise usual course. These tumours often exactly resemble those of mycosis fungoides in clinical appearance, though 


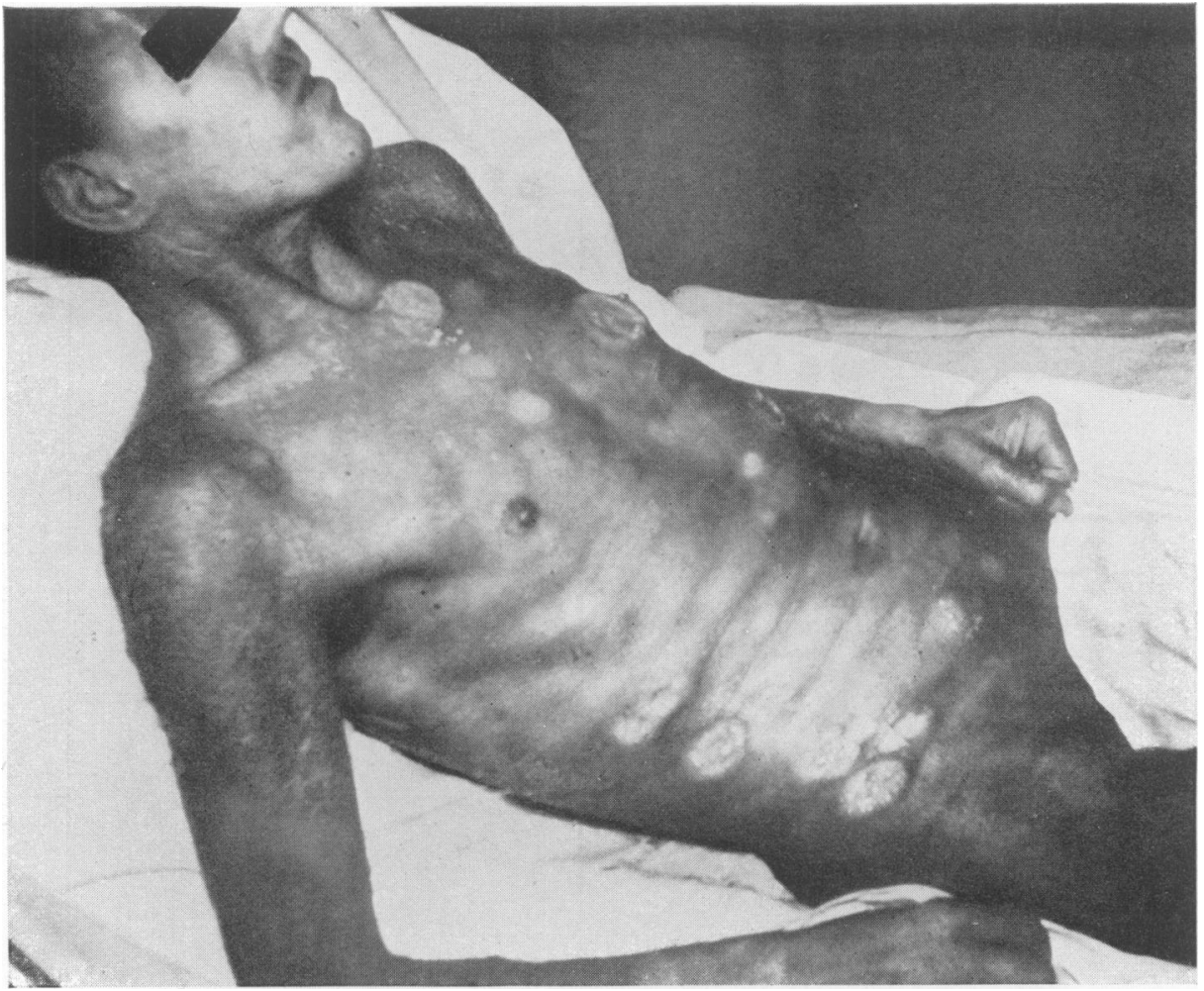

FIG. 3.-Mycosis fungoides. Multiple skin tumours, some showing early ulceration.

they tend to be somewhat harder, they are fewer, and ulceration is less likely to occur. (Fig. 5). Their situation in the substance of the skin, freely mobile over the subcutaneous tissues, their firmness to the touch and reddish or plum colour are characteristic. These features and their rapid growth usually point to the diagnosis, though biopsy is nearly always necessary for the exact classification of the disorder. In the early tumour stage the cell picture may be difficult to interpret and it may be that only later on biopsy of a lymph gland permits accurate diagnosis. This is probably the explanation of at least some of those cases where one reticulosis is said to have been transformed into another.

Similar tumours may occur in the leukaemias. In these cases the masses may be more diffuse and present brawny indurated areas 6 in. or more across. The borders of these infiltrated areas may be ill-defined and, in many respects, the clinical picture resembles that of cellulitis; the purplish colour of the lesion, however, and the complete lack of pain make distinction easy.

In sarcoidosis two types of skin nodule may be produced, both showing the typical histological structure. The lesions known as lupus pernio are of a deep red or cyanotic hue. They form welldefined firm infiltrated areas, raised a little above the surface of the surrounding skin. They tend to remain relatively unchanged for a considerable period, usually showing little tendency to enlarge and little tendency to spontaneous healing. These lesions occur most often about the nose and cheeks and on the fingers. The second type of cutaneous sarcoid takes the form of smaller tumours; these, too, are situated chiefly on the face, ears and hands and consist of brownish raised nodules, sharply outlined and usually $\mathrm{I}$ or $2 \mathrm{~cm}$. in diameter. Their degree of pinkness is variable; if they are examined through a glass slide pressed on the skin so as to expel the blood, they have a brownish-yellow semitranslucent appearance resembling that of tuberculous lupus. While these appearances are clinically suggestive of sarcoidosis, the diagnosis must always depend, as in other reticuloses, on the histological picture and biopsy is always necessary. It should, at the same time, be recalled that the clinical and histological picture of sarcoidosis may be closely mimicked by other disorders; leprosy may produce very similar lesions, while the granulomata 


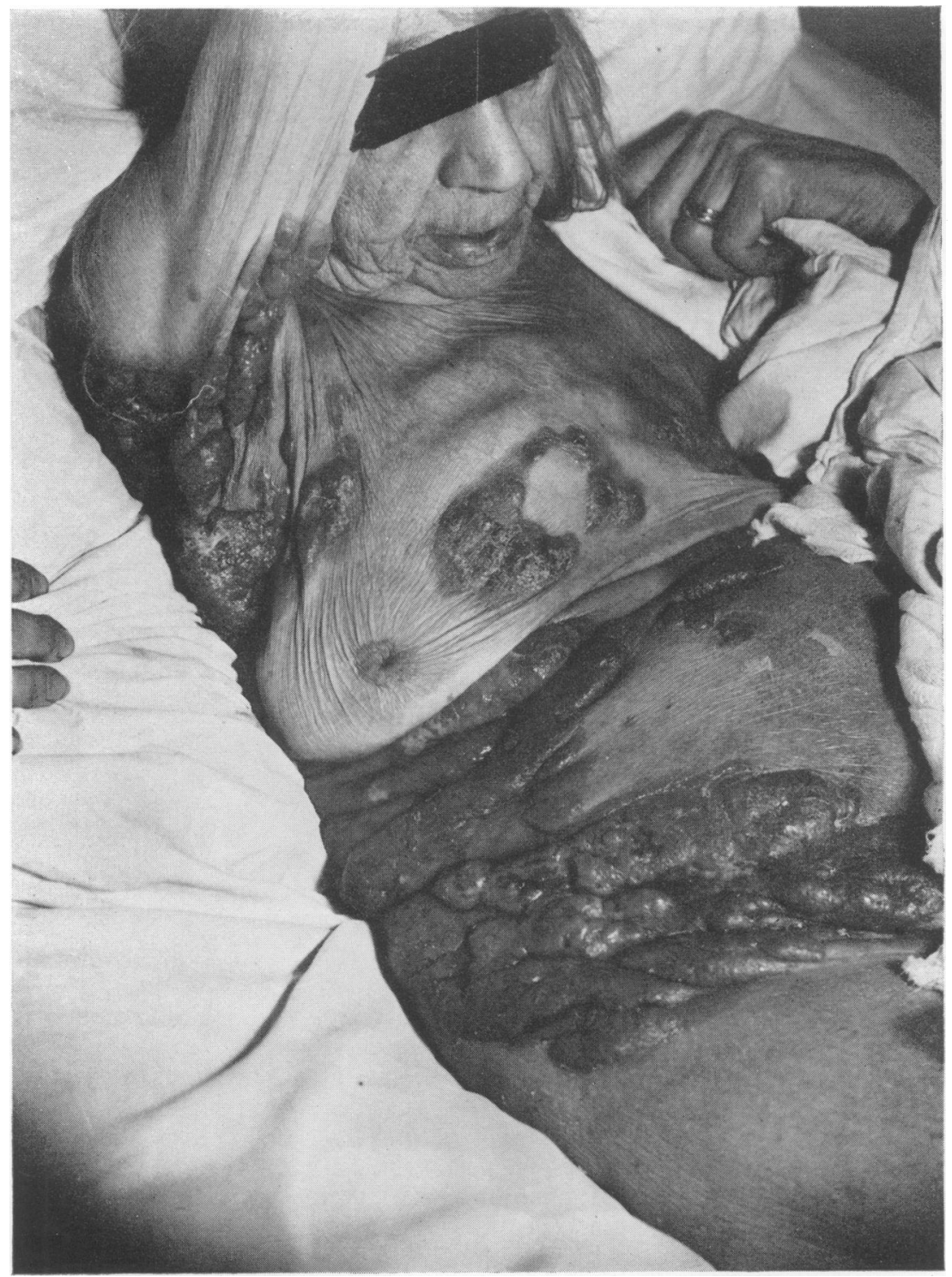

FIG. 4.-Mycosis fungoides. Multiple punched out ulcers, many of which would heal spontaneously after several weeks. The skin of other parts of the body, e.g. shoulders, is diffusely red and scaly.

which form around particles of silica and beryllium in the skin may be distinguishable only by finding the foreign bodies.

The brownish circumscribed tumour of sarcoidosis may easily be confused on clinical examination with a variety of eosinophilic granuloma. The latter term has been variously used to describe lesions in the skin and elsewhere. The localized granuloma now under consideration is so named because it consists of a mixed cellular in- filtrate in which eosinophils are very numerous. These cases do not seem to be produced by any local agent; at the same time, disseminated lesions do not often appear and it is doubtful whether this type of eosinophilic granuloma should be classified with the reticuloses (Rook, 1950).

On close inspection of the sarcoid nodule in the skin it is sometimes possible to distinguish a fine stippling of brownish pin-point nodules. In some cases these nodules are more widely separated. 


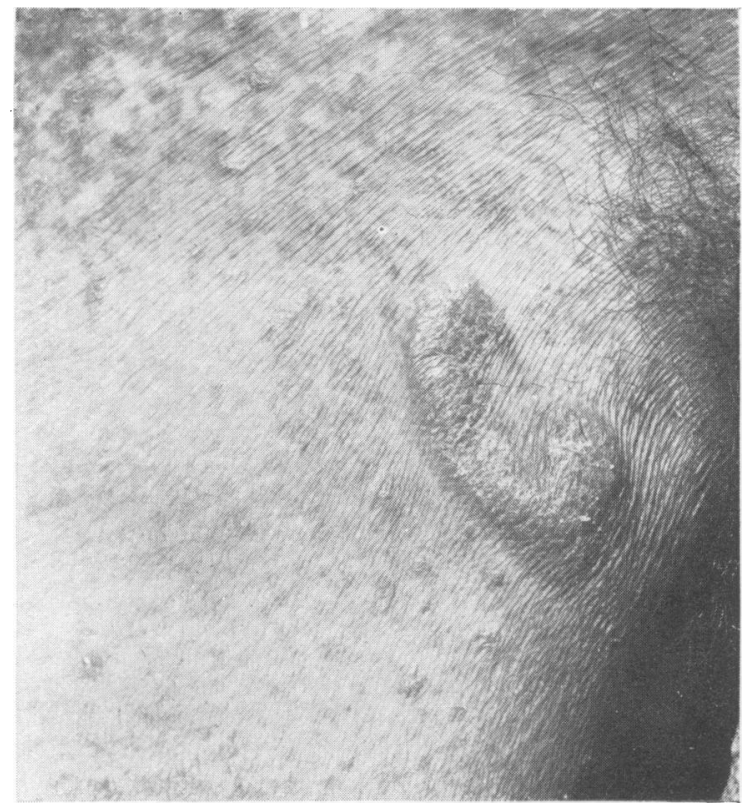

FIG. 5.-Primary tumour of Hodgkin's disease in the skin. This firm elevated plaque was followed by other skin lesions and later by typical systemic involvement (see Fairburn, I950).

thus giving rise to a reddish area, perhaps I or 2 $\mathrm{cm}$. across, in which scattered yellow-brown stipples about $\mathbf{I} \mathrm{mm}$. or less in diameter are scattered. These miliary sarcoid lesions may be widely spread on the skin of the trunk and limbs. Other signs of sarcoidosis may be present in these cases but, nevertheless, the diagnosis must largely depend on histological examination. In rare cases with apparently identical skin lesions, biopsy shows a more mixed cellular infiltrate which, even in the presence of other signs suggestive of sarcoidosis, makes the latter diagnosis unacceptable. The exact classification of this latter type of case is very difficult and is better based on histological examination of the lymph nodes, if these are involved, than on skin biopsy.

Apart from these broad considerations, there are a few clinical dermatoses which have features of their own and which are probably to be considered as reticuloses. Miliary lymphocytoma is one of these (Bettley, 1947). This rare syndrome presents a remarkably constant clinical picture of pinhead size, semi-translucent bluish nodules scattered on the forehead and upper half of the face. Irritation usually affects the trunk and limbs as well as the face and the skin is abnormally sensitive to light. Biopsy shows the facial nodules to be composed of lymphocytic germ centres closely resembling those found in the lymph nodes. It is very doubtful whether benign lymphocytoma ever produces leukaemia or any lesion elsewhere in the reticulo-endothelial system and, for this reason, it is thought by some to be nothing more than a special type of reaction in a person who is abnormally sensitive to light.

Urticaria pigmentosa may be considered in this category; it consists of the widespread development of well-outlined, pigmented circular and oval areas, chiefly on the trunk, which are readily converted into urticarial wheals on slight trauma. Histologically the lesion contains a remarkable number of mast cells and this has led to the suggestion that urticaria pigmentosa should be classified as a reticulosis involving elements of the mast cell series. By others, however, the disorder is regarded as naevoid in nature.

Figs. 2, 3, 4 and 5 were prepared in the Photographic Department of the Middlesex Hospital, London.

\section{BIBLIOGRAPHY}

BETTLEY, F. R. (1947), Brit. F. Derm., 59, 70. FAIRBURN, E. A. (I950), Ibid., 62, 504. GOLDSMITH, W. N. (1944), Ibid., 56, 107.
PAUTRIER, L-M., and WORINGER, F. (1932), Bull. Soc. franc. Derm., 39, 947.

ROOK, A. J. (1950), Brit. f. Derm., 62, 4 II. 